Check for updates

Cite this: RSC Adv., 2017, 7, 29004

\title{
Novel multifunctional dental bonding agent for class- $V$ restorations to inhibit periodontal biofilms
}

\author{
Lin Wang, ${ }^{\text {ab }}$ Chunyan Li, ${ }^{c}$ Michael D. Weir, ${ }^{b}$ Ke Zhang, (D)*bd Yanmin Zhou, ${ }^{* c}$ \\ Hockin H. K. Xu (DD bef and Mark A. Reynolds ${ }^{b}$
}

We recently developed a dental bonding agent to bond restorations to teeth using nanoparticles of amorphous calcium phosphate (NACP) for remineralization with rechargeable calcium and phosphate ion release. The objectives of this study were to: (1) incorporate an antibacterial monomer dimethylaminohexadecyl methacrylate (DMAHDM) and a protein-repellent agent 2-methacryloyloxyethyl phosphorylcholine (MPC); and (2) investigate protein adsorption and periodontitis-related biofilms for the first time. A primer, used to prime tooth structures for bonding, was made with pyromellitic glycerol dimethacrylate (PMGDM) and 2-hydroxyethyl methacrylate (HEMA). An adhesive was made with PMGDM, ethoxylated bisphenol A dimethacrylate and HEMA. NACP, MPC and DMAHDM were incorporated. Streptococcus gordonii, Actinomyces naeslundii, Porphyromonas gingivalis, Fusobacterium nucleatum were cultured to form single and multi-species biofilms. Colony-forming units (CFU), live/dead, metabolic activity, and polysaccharide were measured. Adding DMAHDM, MPC and NACP into the bonding agent did not compromise the dentin bond strength $(p>0.1)$. Bonding agents with 5\% MPC reduced protein adsorption to $1 / 15$ that of the control $(p<$ 0.05). Bonding agents with 5\% DMAHDM + 5\% MPC had much greater reduction in biofilms than DMAHDM or MPC alone $(p<0.05)$. Biofilm CFU was reduced by 3 to 4 log via DMAHDM + MPC. Metabolic activities and polysaccharide of biofilms were also substantially reduced $(p<0.05)$. In conclusion, a novel bonding agent was developed for dental restorations with inhibition of biofilms, reducing CFU by 3 to 4 log. Besides remineralization and acid-neutralization via NACP to inhibit caries as shown previously, the multifunctional adhesive is promising for root restorations with subgingival margins to suppress periodontal pathogens and protect the periodontium.

Received 26th December 2016

Accepted 25th May 2017

DOI: 10.1039/c6ra28711e

rsc.li/rsc-advances subgingival margins, which can hinder cleaning and provide pockets for the growth of periodontitis-related bacteria. ${ }^{3}$ This in turn would lead to the tooth's clinical attachment loss and enhance the development of periodontitis. Current resin-based restorations for class $\mathrm{V}$ restorations usually are not antibacterial. Instead, resins were shown to accumulate more biofilms/plaques than other materials, ${ }^{4}$ which could aggravate the progress of periodontitis.

Subgingival dental biofilms are the principal aetiological factor of periodontitis. ${ }^{5}$ Biofilm growth is initiated by an acquired salivary pellicle formation. In this pellicle, several salivary components including glycoprotein and proline-rich proteins promote the adherence of bacterial cells. The early attached dominant species of bacteria are streptococci and members of the yellow and purple complexes, such as Actinomyces spp., which soon develop a polymicrobial community. ${ }^{6}$ Actinomyces naeslundii (A. naeslundii) represents $27 \%$ of the pioneer strains. ${ }^{7}$ Furthermore, this species is able to adapt to extremely fluctuating conditions, which is necessary for a pioneer bacteria strain to attach to dental surfaces. ${ }^{8}$ In addition, Streptococcus gordonii ( $S$. gordonii) is a well-known early colonizer, which can co-aggregate with a variety of oral bacteria 
and bind to several host molecules. ${ }^{9}$ Members of the red, orange and green complexes colonize as the biofilm matures. ${ }^{6}$ In clinical situations associated with periodontitis, there is a net predominance of orange and red complex species. ${ }^{10}$ Furthermore, Porphyromonas gingivalis ( $P$. gingivalis), as a member of red complex, has been implicated as a major etiologic agent in the development and progression of periodontitis. ${ }^{\mathbf{1 1}}$ It possesses potent virulence factors to neutralize host defense and destroy periodontal tissues, leading to progressive loss of alveolar bone and periapical bone. ${ }^{12}$ Moreover, Fusobacterium nucleatum (F. nucleatum), as a member of orange complex, plays a central role as a bridge bacterium between early and late colonizers and is correlated with increased probing depth and progressive periodontal ligament reduction in periodontitis. ${ }^{13}$ Therefore, these four species were selected to form a multispecies periodontal biofilms in the present study.

Due to their esthetics and direct-filling capabilities, composites are popular for tooth cavity restorations, ${ }^{\mathbf{1 4 - 1 6}}$ and composite restorations are bonded to the tooth structure using bonding agent. ${ }^{17-20}$ Research on dental bonding agents has focused on ways to enhance the longevity and effectiveness of the bonded interface. ${ }^{17-20}$ Research on composites has focused on resin compositions, fillers, and curing properties. The fillers that have been used in dental resins include colloidal silica, glass, hydroxyapatite, ytterbium trifluoride, etc. Fillers have also been incorporated into bonding agents to improve the mechanical properties of the resin, reduce the polymerization shrinkage, and enhance the resin-tooth bond strength. ${ }^{21-23}$ In addition, to develop resins with remineralization capabilities, calcium and phosphate ion-releasing fillers were incorporated into resins. The release of calcium and phosphate ions enhanced the formation of hydroxyapatite and thus remineralized tooth lesions to avoid caries development. ${ }^{24}$ However, traditional calcium phosphate-containing resins had low mechanical strengths. More recently, nanoparticles of amorphous calcium phosphate (NACP) were synthesized and incorporated into resins with substantially improved mechanical properties as well as remineralization and acid-neutralization capabilities. ${ }^{25}$

At the bonded interface, microleakage and bacterial invasion at the margins often lead to secondary caries and restoration failure. $^{26,27}$ Therefore, efforts were made to inhibit cariogenic bacteria and their acid production, in order to reduce recurrent caries. $^{28}$ Quaternary ammonium monomers (QAMs) such as 12-methacryloyloxydodecylpyridinium bromide (MDPB) were copolymerized in resins to yield antibacterial activities via the "contact-inhibition" mechanism. ${ }^{29}$ Recently, bonding agent containing a new monomer dimethylaminohexadecyl methacrylate (DMAHDM) showed a strong antibiofilm activity. ${ }^{30}$

Salivary protein coating on resin could decrease the efficacy of "contact-inhibition". ${ }^{31,32}$ Therefore, efforts were made to develop protein-repellent functions. ${ }^{33}$ 2-Methacryloyloxyethyl phosphorylcholine (MPC) is a methacrylate with phospholipid polar groups and is a common biopolymer. ${ }^{34}$ Polymers containing MPC are known to reduce protein adsorption and bacterial adhesion..$^{35,36}$ In our previous studies, MPC-containing adhesives showed strong protein-repellent properties and inhibited cariogenic bacteria. ${ }^{37,38}$ However, to date, there has been no report on bonding agent containing MPC and
DMAHDM for class $\mathrm{V}$ restorations to inhibit biofilms of periodontal pathogens.

The objectives of this study were to incorporate MPC and DMAHDM into bonding agent for potential use in class $\mathrm{V}$ restorations, and to investigate its effects on multispecies and single species periodontal biofilms for the first time. It was hypothesized that: (1) incorporation of DMAHDM and MPC into bonding agent would not compromise the dentin bond strength; (2) MPC and DMAHDM together would reduce periodontal biofilms much more than MPC or DMAHDM alone; (3) the killing efficacy of DMAHDM-MPC-containing adhesive would be different between multispecies periodontal biofilm and various single-species periodontal biofilms.

\section{Materials and methods}

\subsection{Fabrication of bonding agent containing DMAHDM, MPC and NACP}

The experimental primer consisted of pyromellitic dianhydride glycerol dimethacrylate (PMGDM, Esstech, Essington, PA) and 2hydroxyethyl methacrylate (HEMA, Esstech) at a mass ratio $10: 3$, with $50 \%$ acetone solvent (all by mass), following a previous study. ${ }^{39}$ The experimental adhesive contained PMGDM, ethoxylated bisphenol A dimethacrylate (EBPADMA, Sigma-Aldrich, St. Louis, MO), HEMA and bisphenol A glycerolate dimethacrylate (BisGMA, Esstech) at 45/40/10/5 ratio (referred to as PEHB). ${ }^{40}$ As the photo-initiator, $1 \%$ phenyl bis(2,4,6-trimethylbenzoyl)phosphine oxide (BAPO, Sigma-Aldrich) was added to the adhesive. This bonding system was used because a previous study showed that the incorporation of nanoparticles of amorphous calcium phosphate (NACP) yielded substantial release and recharge of calcium (Ca) and phosphate (P) ions. ${ }^{40}$

NACP were incorporated into adhesive resin, because previous studies showed that NACP released $\mathrm{Ca}$ and $\mathrm{P}$ ions and remineralized tooth lesion. ${ }^{\mathbf{4 1 , 4 2}}$ NACP were synthesized using a spray-drying technique as previously described. ${ }^{43}$ Briefly, calcium carbonate and dicalcium phosphate were dissolved in acetic acid to produce $\mathrm{Ca}$ and $\mathrm{P}$ concentrations of 8 and $5.333 \mathrm{mmol} \mathrm{L}^{-1}$, respectively, thus yielding a $\mathrm{Ca} / \mathrm{P}$ molar ratio of 1.5 , the same as that for ACP $\left[\mathrm{Ca}_{3}\left(\mathrm{PO}_{4}\right)_{2}\right]$. This solution was sprayed into a heated chamber, and an electrostatic precipitator collected the dried particles. This method produced NACP with a mean particle size of $116 \mathrm{~nm} \cdot{ }^{43}$ NACP were incorporated into adhesive resin at a filler level of $30 \%$ by mass, following a previous study on ion release and recharge. ${ }^{40}$

MPC was obtained commercially (Sigma-Aldrich) which was synthesized via a method reported previously. ${ }^{34}$ MPC powder was mixed into primer at MPC/(primer + MPC) mass fractions of $0 \%, 2.5 \%, 5 \%$, and $7.5 \%$. Similarly, MPC was mixed into adhesive at MPC/(adhesive + MPC) mass fractions of $0 \%, 2.5 \%$, $5 \%$, and $7.5 \%$. MPC at more than $7.5 \%$ was not used due to a decrease in dentin bonding strength in preliminary studies.

DMAHDM was synthesized using a Menschutkin reaction in which a tertiary amine group was reacted with an organohalide. ${ }^{\mathbf{4 4}}$ Briefly, $10 \mathrm{mmol}$ of 2-(dimethylamino)ethyl methacrylate (DMAEMA, Sigma-Aldrich) and $10 \mathrm{mmol}$ of 1-bromohexadecane (BHD, TCI America, Portland, OR) were combined with $3 \mathrm{~g}$ of 
ethanol in a $20 \mathrm{~mL}$ scintillation vial. The vial was stirred at $70{ }^{\circ} \mathrm{C}$ for $24 \mathrm{~h}$. The solvent was removed via evaporation, yielding DMAHDM as a clear, colorless, and viscous liquid. ${ }^{45}$ DMAHDM was mixed with primer and adhesive at a DMAHDM mass fraction of $5 \%$, following a previous study. ${ }^{38}$

\subsection{Dentin shear bond strength testing}

Ten groups were tested for dentin shear bond strength using extracted human teeth:

(1) Prime \& Bond NT (Caulk/Dentsply, Milford, DE) (denoted commercial control);

(2) Experimental primer and adhesive control (denoted PEHB control);

(3) Experimental primer; adhesive was filled with 30\% NACP (denoted PEHB-NACP);

(4) Experimental primer $+5 \%$ DMAHDM; adhesive $+5 \%$ DMAHDM + 30\% NACP (denoted 5DMAHDM);

(5) Experimental primer $+2.5 \% \mathrm{MPC}$; adhesive $+2.5 \% \mathrm{MPC}+$ $30 \%$ NACP (denoted PEHB $+2.5 \mathrm{MPC}$ );

(6) Experimental primer $+5 \%$ MPC; adhesive $+5 \% \mathrm{MPC}+$ $30 \%$ NACP (denoted 5MPC);

(7) Experimental primer $+7.5 \% \mathrm{MPC}$; adhesive $+7.5 \% \mathrm{MPC}+$ $30 \%$ NACP (denoted 7.5MPC);

(8) Experimental primer $+5 \%$ DMAHDM $+2.5 \%$ MPC; adhesive $+5 \%$ DMAHDM $+2.5 \%$ MPC $+30 \%$ NACP (denoted 5DMAHDM + 2.5MPC);

(9) Experimental primer + 5\% DMAHDM + 5\% MPC; adhesive $+5 \%$ DMAHDM $+5 \%$ MPC $+30 \%$ NACP (denoted 5DMAHDM + 5MPC);

(10) Experimental primer + 5\% DMAHDM + 7.5\% MPC; adhesive $+5 \%$ DMAHDM $+7.5 \%$ MPC $+30 \%$ NACP (denoted $5 \mathrm{DMAHDM}+7.5 \mathrm{MPC})$.

The commercial control (1) was included for comparative purpose for dentin shear bond strength. According to the manufacturer, Prime \& Bond NT was a total-etching one-bottle bonding system with $30 \%$ methacrylates and less than $10 \%$ methyl methacrylate, which used acetone as solvent (60\%). The experimental control (2) also used acetone as solvent. For dentin bond test, extracted human third molars were used, and all experiments were performed in compliance with the relevant laws and institutional guidelines of United States. The protocol was approved by the University of Maryland Institutional Review Board, and the informed consent was obtained from all human subjects. The each tooth was cut to remove the crown under water coolant (Isomet, Buehler, Lake Bluff, IL), then ground on 600-grit SiC paper until occlusal enamel was completely removed. Dentin was etched with 37\% phosphoric acid for $15 \mathrm{~s}$. The etched dentin was brushed with primer, and the solvent was evaporated with an air stream. An adhesive was applied and light-cured for $10 \mathrm{~s}$ using a quartz-tungsten-halogen lightcuring unit with $600 \mathrm{~mW} \mathrm{~cm}^{-2}$ (Demetron VCL401, Demetron, CA). A stainless-steel iris mold with $4 \mathrm{~mm}$ diameter and $1.5 \mathrm{~mm}$ thickness was placed onto the adhesive-treated dentin surface, filled with a composite (TPH, Caulk/Dentsply), and light-cured for $60 \mathrm{~s}$ (Demetron VCL401). ${ }^{46}$ The bonded specimens were stored in distilled water at $37^{\circ} \mathrm{C}$ for $24 \mathrm{~h}$. A chisel on a Universal Testing Machine (MTS, Eden Prairie, MN) was aligned to be parallel to the composite-dentin interface. ${ }^{46} \mathrm{Load}$ was applied at a cross-head of $0.5 \mathrm{~mm} \mathrm{~min}^{-1}$ until the bond failed. Dentin shear bond strength $S=4 P /\left(\pi d^{2}\right)$, where $P$ is the load at failure, and $d$ is the diameter of the composite. ${ }^{46}$

\subsection{Specimen fabrication for protein adsorption and biofilm experiments}

The dentin bond strength results showed that bonding agent 5DMAHDM + 7.5MPC had a lower bond strength; therefore, it was not included in the subsequent protein-repellent test. In addition, preliminary study showed that $2.5 \%$ MPC was not as effective as 5\% MPC to repel proteins. Hence, five bonding agents (the aforementioned groups 1, 3, 4, 6 and 9) were tested in protein adsorption and biofilm experiments.

Resin disks were made using the cover of a 96-well plate (Costar, Corning, NY, USA) as molds following a previous study. ${ }^{47}$ Ten $\mu \mathrm{L}$ of primer was placed at the bottom of each dent in the 96-well plate. After drying with air, $20 \mu \mathrm{L}$ of adhesive was placed into the dent and photo-cured for $30 \mathrm{~s}$ (Demetron VCL401), using a mylar strip covering to obtain a disk of approximately $8 \mathrm{~mm}$ in diameter and $0.5 \mathrm{~mm}$ in thickness. The cured disks were immersed in water and stirred with a magnetic bar at $100 \mathrm{rpm}$ for $1 \mathrm{~h}$ to remove any uncured monomers, following a previous study. ${ }^{\mathbf{2 6}}$ The disks were sterilized with ethylene oxide (AnproleneAN 74i, Andersen, Haw River, NC, USA) and de-gassed for 7 days.

\subsection{Protein adsorption assay}

Protein adsorbed on resin disks was determined using a micro bicinchoninic acid (BCA) method. ${ }^{36,48}$ Thirty resin disks were fabricated with six disks for each group. Each disk was immersed in phosphate buffered saline (PBS) for $2 \mathrm{~h}$, and then immersed in $4.5 \mathrm{~g} \mathrm{~L}^{-1}$ bovine serum albumin (BSA, Sigma-Aldrich) solution at $37^{\circ} \mathrm{C}$ for $2 \mathrm{~h} .{ }^{36,48}$ The disks were rinsed with PBS, then immersed in $1 \%$ sodium dodecylsulfate (SDS) in PBS and sonicated for $20 \mathrm{~min}$ to detach the BSA adsorbed on the disk. ${ }^{36,48}$ A protein analysis kit (micro BCA assay, Fisher Scientific, Pittsburgh, PA, USA) was used to determine the BSA concentration in the SDS solution. $25 \mu \mathrm{L}$ of the SDS solution and $200 \mu \mathrm{L}$ of BCA were mixed into the wells of a $96-w e l l$ plate and incubated at $60^{\circ} \mathrm{C}$ for $30 \mathrm{~min}$. The absorbance at $562 \mathrm{~nm}$ was measured via a microplate reader (SpectraMax M5, Molecular Devices, Sunnyvale, CA, USA). Standard curves were prepared using the BSA standard. ${ }^{36,48}$

\subsection{Periodontal bacteria strains and culture media}

The use of all bacterial species was approved by University of Maryland Baltimore Institutional Review Board. All four species were obtained from the American Type Culture Collection (ATCC, Manassas, VA, USA): S. gordonii ATCC10558; A. naeslundii ATCC12104; P. gingivalis ATCC33277; F. nucleatum ATCC25586.

$S$. gordonii were grown in brain-heart infusion broth (BHI, Sigma-Aldrich) at $37{ }^{\circ} \mathrm{C}$ aerobically (95\% air, 5\% $\mathrm{CO}_{2}$ ), following ACTT's instructions. A. naeslundii, $P$. gingivalis and $F$. nucleatum were grown in tryptic soy broth (TSB, Sigma-Aldrich) supplemented with yeast extract $\left(5 \mathrm{~g} \mathrm{~L}^{-1}\right)$, L-cysteine hydrochloride 
$\left(0.5 \mathrm{~g} \mathrm{~L}^{-1}\right)$, hemin $\left(5 \mathrm{mg} \mathrm{L}^{-1}\right)$ and menadione $\left(1 \mathrm{mg} \mathrm{L}^{-1}\right)$ at $37^{\circ} \mathrm{C}$ anaerobically $\left(90 \% \mathrm{~N}_{2}, 5 \% \mathrm{CO}_{2}, 5 \% \mathrm{H}_{2}\right)$, following previous studies. ${ }^{\mathbf{1 1 , 4 9}}$ For each species, the inoculum was adjusted to $10^{8}$ colony-forming unit counts $\mathrm{CFU} \mathrm{mL}^{-1}$ for biofilm formation, based on the standard curve of $\mathrm{OD}_{600 \mathrm{~nm}} v s$. CFU mL $\mathrm{mL}^{-1}$ for each species. ${ }^{49}$

\subsection{Multi-species and single species periodontal biofilms on adhesive disks}

The five bonding agents (groups 1, 3, 4, 6 and 9) were tested with multispecies periodontal biofilms. Then, two bonding agents (groups 3 and 9) were selected to test the four single-species biofilms to compare the killing efficacy between single-species and multispecies periodontal biofilms.

Saliva was collected from fifteen healthy adult donors without caries, periopathology and endodontic infections, and without the use of antibiotics in the last three months. The donors did not brush teeth for $24 \mathrm{~h}$ and abstained from food and drink intake for $2 \mathrm{~h}$ prior to saliva donation. An equal volume of saliva from each of the fifteen donors was combined together. Saliva was centrifuged at $3000 \mathrm{rpm}$ for $20 \mathrm{~min}$ to remove cellular debris. The supernatant was collected and filtersterilized through sterile $0.22 \mu \mathrm{m}$ filter (VWR International, Radnor, PA, USA). The sterile saliva was used to coat a salivary pellicle on the resin disks by immersing each disk in sterile saliva in a 24 -well plate for $4 \mathrm{~h}$ at $37{ }^{\circ} \mathrm{C} .{ }^{50}$

A multispecies biofilm was formed following a previous study. ${ }^{51} S$. gordonii, A. naeslundii, $F$. nucleatum and $P$. gingivalis were selected to develop a periodontal four-species biofilm. ${ }^{\mathbf{2}}$ Briefly, $250 \mu \mathrm{L}$ of $S$. gordonii suspension, $500 \mu \mathrm{L}$ of $A$. naeslundii suspension, and $1000 \mu \mathrm{L}$ of $F$. nucleatum and $P$. gingivalis suspensions were mixed and pipetted into $50 \mathrm{~mL}$ TSB supplemented medium. ${ }^{51}$ The sterile saliva pellicle-coated resin disks were transferred to a new 24-well plate. Each well was inoculated with $1.5 \mathrm{~mL}$ mixed bacterial suspension and incubated in anaerobic conditions $\left(90 \% \mathrm{~N}_{2}, 5 \% \mathrm{CO}_{2}, 5 \% \mathrm{H}_{2}\right)$ at $37^{\circ} \mathrm{C}$ for $72 \mathrm{~h}$, and the medium was changed every 24 h. ${ }^{53}$ Plates containing only culture medium were also incubated to check for sterility. This totaled 3 days of culture, which was shown to form periodontal biofilms on resins. ${ }^{53}$

Single species biofilms were developed as described before. ${ }^{49}$ $S$. gordonii biofilm was grown in BHI broth aerobically, while the other three types of single-species biofilms were grown in TSBsupplemented broth anaerobically. The salivary pellicle-coated adhesive disks were transferred to a new 24-well plate, and each bacterial species was inoculated at a concentration of $10^{7}$ CFU $\mathrm{mL}^{-1}$ in $1.5 \mathrm{~mL}$ medium in each well. After every $24 \mathrm{~h}$, disks with adherent biofilms were transferred to new 24-well plates with fresh medium. Three-day single-species biofilms were formed to be consistent with multispecies periodontal biofilms.

\subsection{Live/dead bacteria imaging}

Each resin disk with 3 day biofilm was washed with cysteine peptone water (CPW) to remove the non-adherent bacteria. Live/ dead bacterial kit (Molecular Probes, Eugene, OR) was used following the manufacturer's instructions. A mixture of $2.5 \mu \mathrm{M}$ SYTO 9 and $2.5 \mu \mathrm{M}$ propidium iodide was used to stain each sample for $15 \mathrm{~min}$ in the dark room. Live bacteria were stained with SYTO 9 to emit a green fluorescence. Bacteria with compromised membranes were stained with propidium iodide to emit a red fluorescence. Biofilms were imaged with an inverted epifluorescence microscope (TE2000-S, Nikon, Melville, NY). Three resin disks were tested for each bonding agent with each biofilm, using a total of 15 disks for multispecies biofilm and 24 disks for four single-species biofilms for live/ dead staining. Five random images were taken for each disk, yielding 15 images for each bonding agent with each type of single or multi-species biofilm.

\subsection{Colony-forming unit (CFU) counts}

Six resin disks were made for each bonding agent with each type of biofilms, using a total of 30 disks for multispecies biofilm and 48 disks for four single-species biofilms for CFU counting. Biofilms were formed by culturing for 3 days as described above. Disks were transferred into vials with $2 \mathrm{~mL} \mathrm{CPW}$, and the biofilms were harvested by scraping and sonication/vortexing (Fisher, Pittsburg, PA, USA). Tryptic soy blood agar plates (supplemented with $5 \mathrm{~g} \mathrm{~L}^{-1}$ yeast extract, $0.5 \mathrm{~g} \mathrm{~L}^{-1}$ L-cysteine hydrochloride, $5 \mathrm{mg} \mathrm{L}^{-1}$ hemin, $1 \mathrm{mg} \mathrm{L}^{-1}$ menadione, $5 \%$ sheep blood) were used. Biofilm suspensions were serially diluted, spread onto agar plate and incubated at $37^{\circ} \mathrm{C}$ anaerobically for all biofilms except $S$. gordonii (aerobically), for $72 \mathrm{~h} .{ }^{54}$ Then, the number of colonies was counted by a colony counter (Reichert, NY, USA), which was used with the dilution factor to calculate CFU counts. ${ }^{30}$

\subsection{MTT metabolic assay}

Six resin disks were made for each bonding agent with each type of biofilm, using a total of 30 disks for multispecies and 48 disks for the four single-species biofilms for MTT assay. The MTT assay is a colorimetric assay that relies on the enzymatic reduction of a yellow tetrazolium salt, 3-(4,5-dimethylthiazol-2-yl)-2,5diphenyltetrazolium bromide (MTT), which forms a purple formazan crystal in metabolically active cells. ${ }^{37}$ Disks with 3 day biofilms were transferred into new 24-well plates with $1 \mathrm{~mL}$ of MTT dye $\left(0.5 \mathrm{mg} \mathrm{mL}^{-1} \mathrm{MTT}\right.$ in PBS) in each well and incubated at $37^{\circ} \mathrm{C}$ in $5 \% \mathrm{CO}_{2}$ for $1 \mathrm{~h}$. The disks were then transferred to new 24-well plates, and $1 \mathrm{~mL}$ of dimethyl sulfoxide (DMSO) was added to each well to solubilize the formazan crystals. ${ }^{37}$ The plates were incubated for 20 min with gentle mixing at room temperature in the dark. After mixing via pipetting, $200 \mu \mathrm{L}$ of the DMSO solution was transferred to a 96-well plate, and the absorbance at $\mathrm{OD}_{540 \mathrm{~nm}}$ was measured via the microplate reader (SpectraMax M5). A higher absorbance is related to a higher metabolic activity in the biofilm on the disks. ${ }^{37}$

\subsection{Measurement of polysaccharide production by periodontal biofilms on resins}

For polysaccharide measurement, six resin disks were made for each bonding agent with different biofilms, using a total of 30 disks for multispecies and 48 disks for the four single-species 
biofilms. The water-insoluble polysaccharide in the extracellular polymeric substance (EPS) of biofilms was determined using a phenol-sulfuric acid method. ${ }^{49}$ Each disk with 3 day biofilm was immersed in a vial with $2 \mathrm{~mL} \mathrm{CPW}$, and the biofilm was collected by sonication/vortexing. Centrifugation yielded a precipitate, which was rinsed with PBS and resuspended in 1 $\mathrm{mL}$ of de-ionized water. Then, $1 \mathrm{~mL}$ of $6 \%$ phenol solution was added to the vial, followed by $5 \mathrm{~mL}$ of $95-97 \%$ sulfuric acid. ${ }^{49}$ The vial was incubated for $30 \mathrm{~min}$. Then, $100 \mu \mathrm{L}$ of the solution was transferred into a 96-well plate. The amount of polysaccharide in biofilms was determined by measuring the absorbance at $\mathrm{OD}_{490 \mathrm{~nm}}$ with the microplate reader. Five glucose concentrations of $0,5,10,20,50$ and $100 \mathrm{mg} \mathrm{L}^{-1}$ were used as standard in the conversion of OD readings to polysaccharide concentrations. $^{49}$

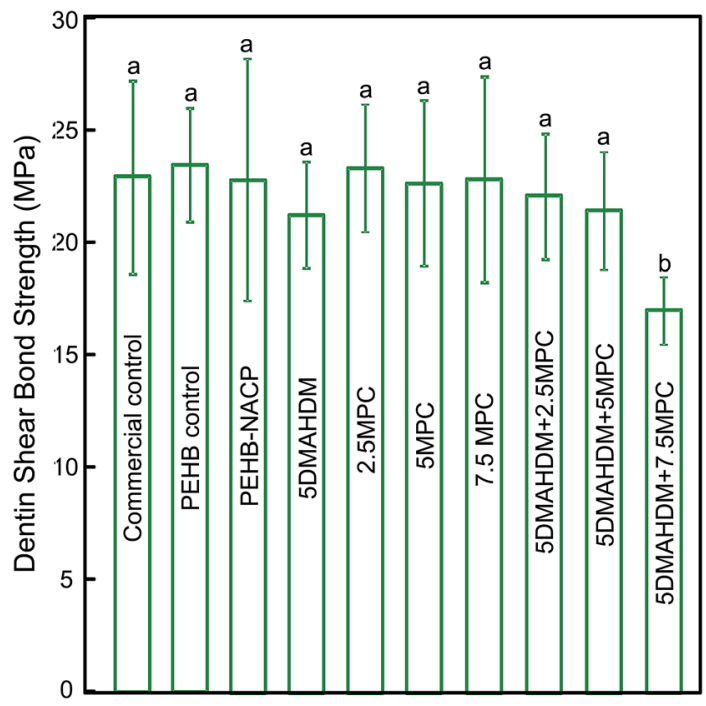

Fig. 1 Dentin shear bond strength using extracted human teeth (mean $\pm \mathrm{sd} ; n=10$ ). Dissimilar letters indicate values that are significantly different from each other $(p<0.05)$.

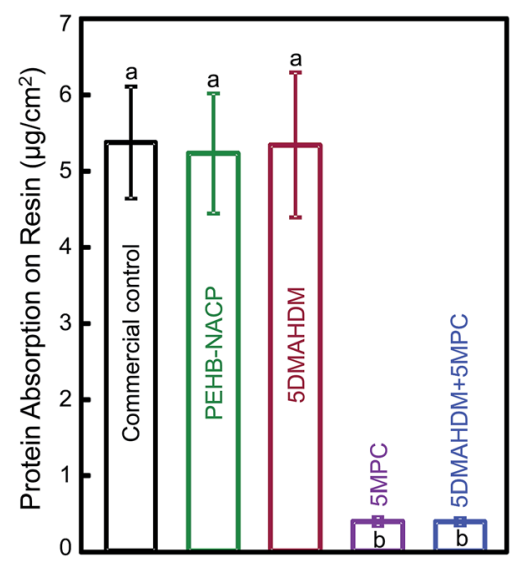

Fig. 2 Protein adsorption onto adhesive resin disk surfaces (mean \pm sd; $n=6$ ). The bonding agent with 5\% DMAHDM + 5\% MPC had much less protein adsorption, which was about 1/15 that of commercial control $(p<0.05)$. Bars with dissimilar letters are significantly different from each other $(p<0.05)$.

\section{Periodontal multi-species biofilm}
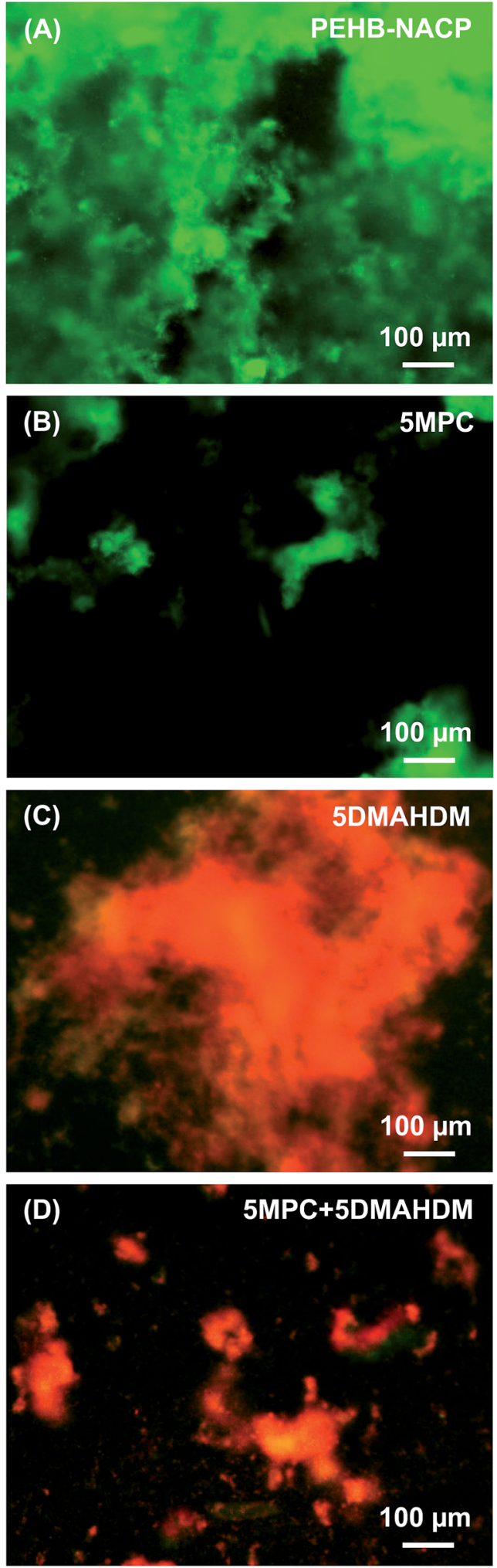

Fig. 3 Representative live/dead images of 3 day multi-species periodontal biofilms on the four adhesive resins: (A) PEHB control, (B) PEHB + 5MPC, (C) PEHB + 5DMAHDM, and (D) 5DMAHDM + 5MPC. Live bacteria were stained green. Dead bacteria were stained red. Resins without DMAHDM had primarily live bacteria. 5DMAHDM + $5 \mathrm{MPC}$ had much less bacterial adhesion, and the biofilms consisted of primarily dead bacteria. 

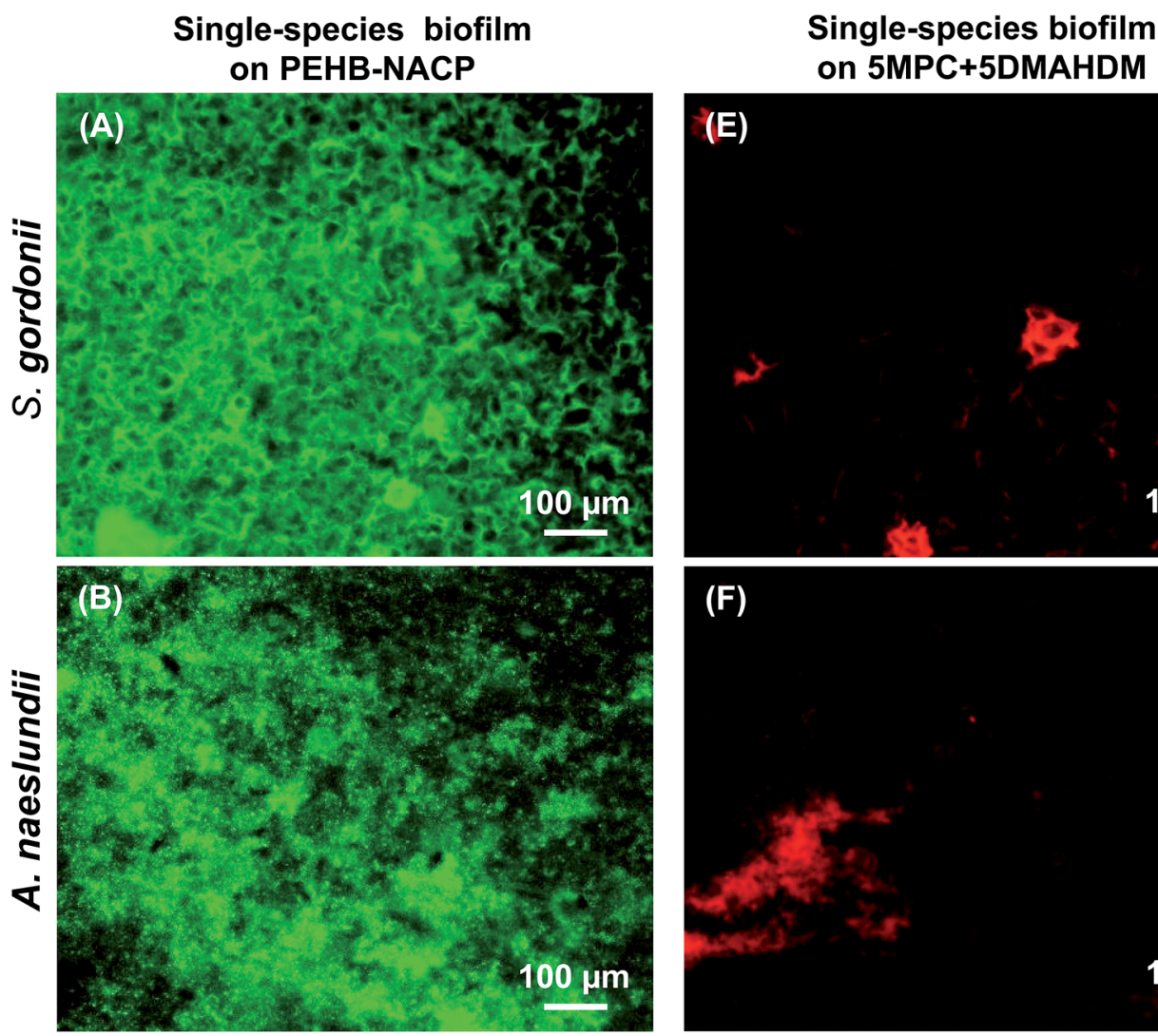

(E) on 5MPC+5DMAHDM
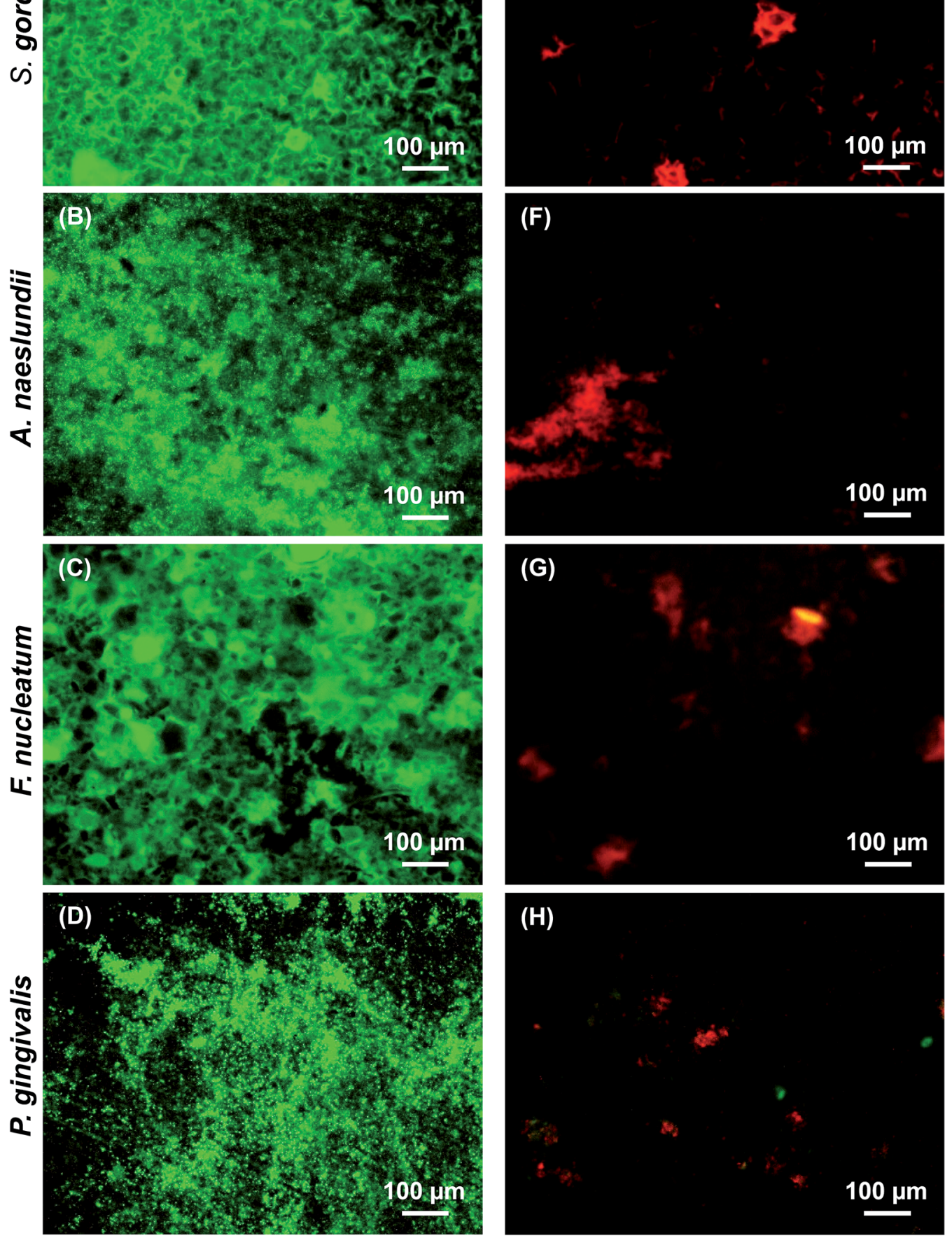

Fig. 4 Representative live/dead images of 3 day single-species periodontal biofilms on two adhesives: (A-D) PEHB-NACP, and (E-H) 5DMAHDM + 5MPC. Live bacteria were stained green. Dead bacteria were stained red. PEHB-NACP had primarily live bacteria. 5DMAHDM + $5 \mathrm{MPC}$ had much less bacteria coverage, and the biofilms consisted of primarily dead bacteria. The species names are listed on the left side. 


\subsection{Statistical analysis}

All data were checked for normal distribution with the Kolmogorov-Smirnov test. One-way analysis of variance (ANOVA) was performed to evaluate differences in dentin bond strength. Twoway ANOVA was used to assess differences in different kinds of biofilms and resin disks. Post hoc multiple comparisons were performed using Tukey's honestly significant difference test. Statistical analyses were performed by SPSS 19.0 software (SPSS, Chicago, IL, USA) at alpha of 0.05 .

\section{Results}

The dentin shear bond strengths are plotted in Fig. 1 (mean \pm sd; $n=6$ ). The first nine groups had similar dentin bond
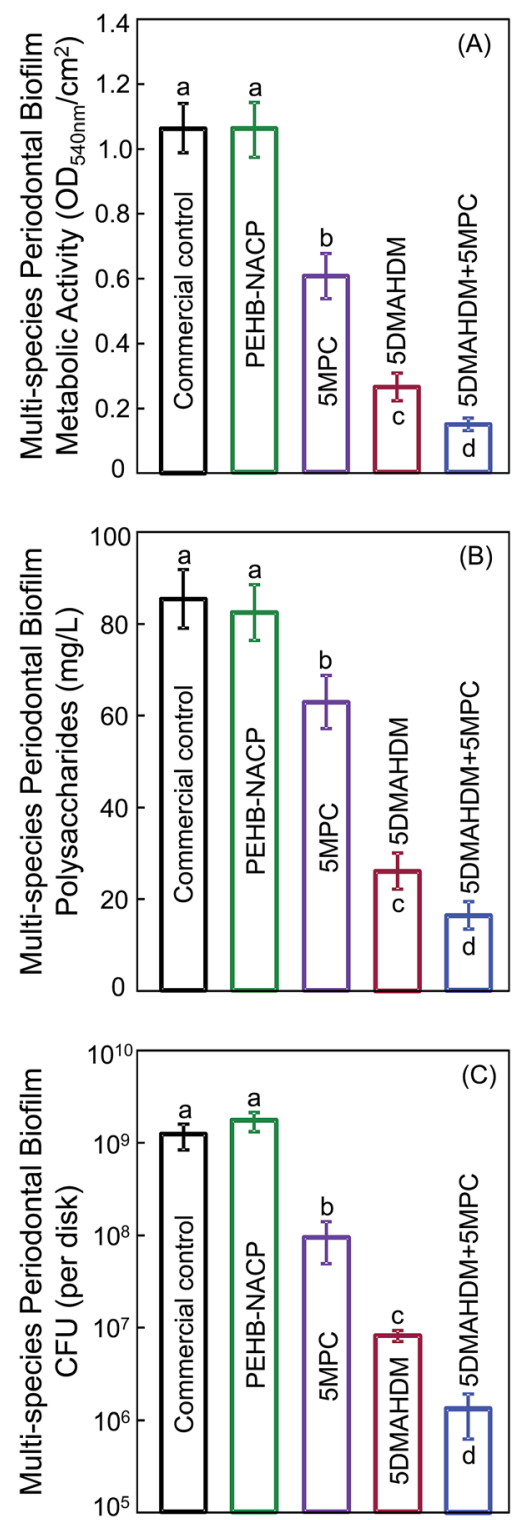

Fig. 5 Multi-species periodontal biofilms on different adhesive disks: (A) metabolic activity, (B) polysaccharide, and (C) CFU (mean $\pm \mathrm{sd} ; n=$ 6). Note log scale for $y$-axis in (C). Bars with dissimilar letters are significantly different from each other $(p<0.05)$. strengths $(p>0.1)$. Adding $30 \%$ NACP into adhesive did not compromise dentin bond strength, compared to PEHB control. Adding 5\% DMAHDM + 5\% MPC into both primer and adhesive did not adversely affect the dentin bond strength, compared to PEHB-NACP $(p>0.1)$. However, adding 5\% DMAHDM $+7.5 \%$ MPC significantly lowered the bond strengths $(p<0.05)$. It should be noted that groups 2-10 all contained NACP at 30\% filler mass fraction in the adhesive.

Fig. 2 plots protein adsorption on resin surfaces (mean $\pm \mathrm{sd}$; $n=6$ ). Adding 5\% DMAHDM had no effect on protein adsorption. Adding 5\% MPC greatly reduced the protein adsorption, compared to PEHB control $(p<0.05)$. The 5\% DMAHDM $+5 \%$ MPC resin had protein adsorption about 15-fold less than that of PEHB control $(p<0.05)$.

Representative live/dead images of 3 day multispecies periodontal biofilms are shown in Fig. 3 on the four groups of bonding agent resin disks. Commercial control had images
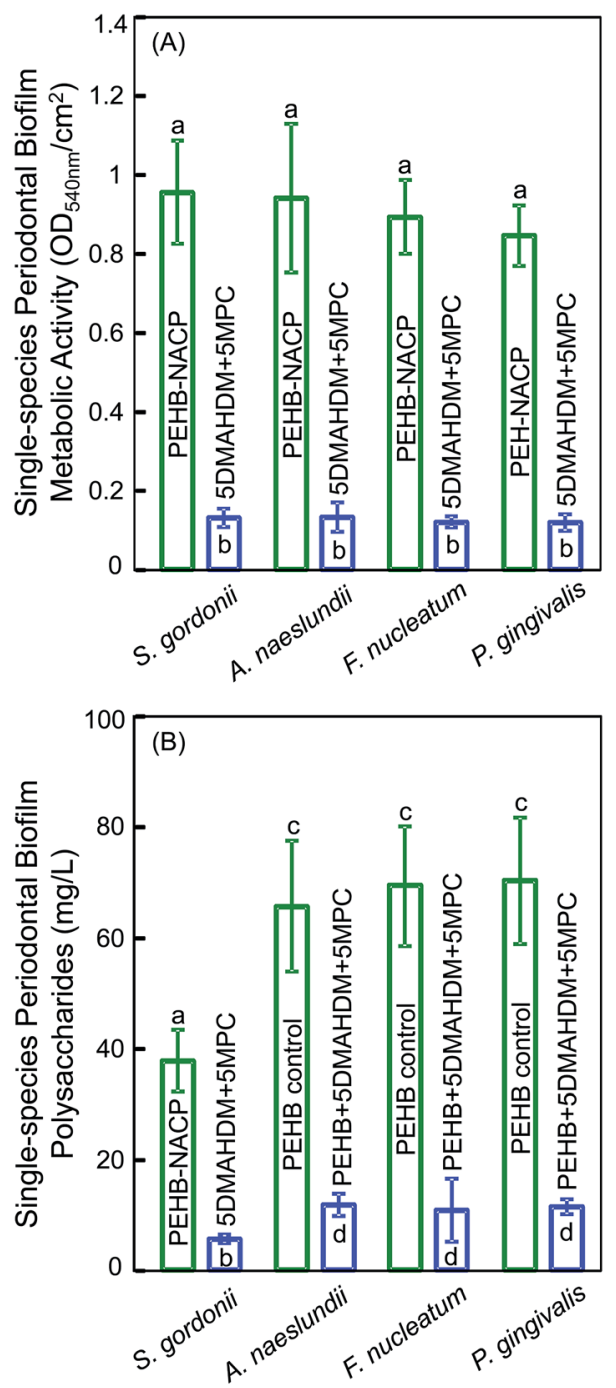

Fig. 6 The four single-species biofilms on PEHB-NACP and 5DMAHDM + 5MPC adhesive resin disks: (A) metabolic activity, and (B) polysaccharide synthesis (mean $\pm \mathrm{sd} ; n=6$ ). Dissimilar letters in each plot indicate significantly different values $(p<0.05)$. 
similar to (A) and was omitted to save space. Live bacteria were stained green, and bacteria with compromised membranes were stained red. Both PEHB-NACP and commercial control were nearly completely covered by live bacteria. In contrast, bonding agents with 5\% MPC had much less bacterial adhesion. Bonding agents with 5\% DMAHDM had substantial amounts of dead bacteria. Bonding agents with 5\% DMAHDM + $5 \%$ MPC had much less bacterial adhesion, and the bacteria were mostly dead.

Single species biofilm live/dead images are shown in Fig. 4. The resin type is listed on the top, and the biofilm species is listed on the left side. PEHB-NACP bonding agents were nearly completely covered by live bacteria. 5DMAHDM +5 MPC had much less bacterial adhesion, and the bacteria were mostly dead.

Fig. 5 plots for multispecies biofilms: (A) the metabolic activity, (B) polysaccharide production, and (C) CFU (mean \pm sd; $n=6)$. Commercial control and PEHB-NACP had similar values $(p>0.1)$. Incorporation of DMAHDM or MPC alone greatly reduced the metabolic activity, polysaccharide and CFU, compared to controls $(p<0.05)$. 5DMAHDM +5 MPC had the least metabolic activity, polysaccharide and CFU $(p<0.05)$. The multispecies biofilm CFU on PEHB control and PEHB + 5 DMAHDM + 5MPC were $1.1 \times 10^{9}$ and $1.2 \times 10^{6}$, respectively, representing a reduction of 3 orders of magnitude $(3 \mathrm{log})$. The $\mathrm{CFU}_{\text {5DMAHDM+5MPC }} / \mathrm{CFU}_{\text {PEHB-NACP }}$ ratio was 0.0010 .

The single-species biofilm metabolic activity and polysaccharide results are plotted in Fig. 6 (mean $\pm \mathrm{sd} ; n=6$ ). The four single-species biofilms on 5DMAHDM + 5MPC had much lower metabolic activity and polysaccharide than PEHB-NACP $(p<0.05)$.

Single-species biofilm CFU results are plotted in Fig. 7 (mean $\pm \mathrm{sd} ; n=6$ ). For all four single-species biofilms, the CFU reduction was about $3 \log$. For $S$. gordonii, A. naelunii, $F$.

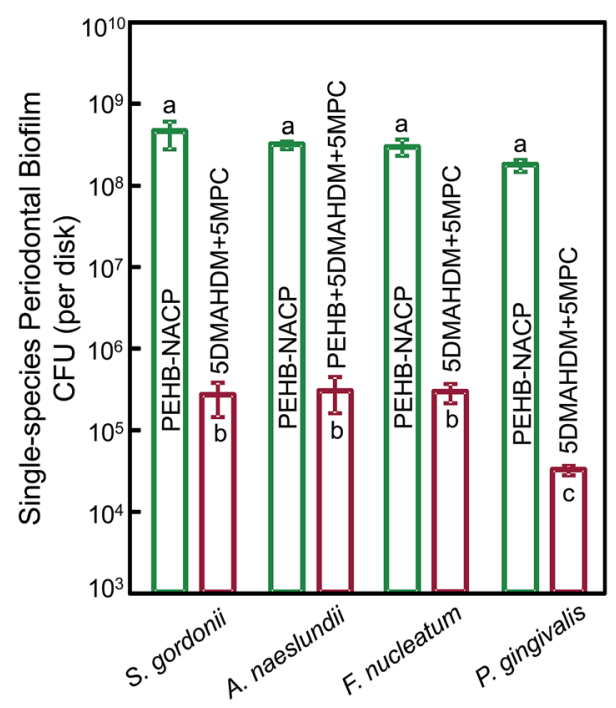

Fig. 7 CFU of the four single-species biofilms on PEHB-NACP and $5 \mathrm{DMAHDM}+5 \mathrm{MPC}$ adhesive resin disks (mean $\pm \mathrm{sd} ; n=6$ ). Note the log scale for the $y$-axis. Bars with dissimilar letters are significantly different from each other $(p<0.05)$. nucleatum and $P$. gingivalis single-species biofilms, the $\mathrm{CFU}_{\mathrm{PEHB}+5 \mathrm{MPC}+5 \mathrm{DMAHDM}} / \mathrm{CFU}_{\mathrm{PEHB}}$ control ratios were: 0.0006, $0.0009,0.0009$ and 0.0002 , respectively.

\section{Discussion}

The present study developed a novel bioactive multifunctional bonding agent for class $\mathrm{V}$ restorations. It contained DMAHDM and MPC to inhibit periodontal multi-species biofilms and NACP for remineralization. The hypotheses were proven that adding MPC and DMAHDM did not compromise the dentin bond strength; that DMAHDM + MPC exerted a much greater anti-biofilm function against periodontal biofilms than MPC or DMAHDM alone. In addition, the killing efficacy of DMAHDMMPC bonding agent was slightly different against multi-species and the four different single-species periodontal biofilms.

Periodontal disease is one of the two most prevalent oral diseases contributing to the global burden of chronic diseases. ${ }^{55}$ It is widespread worldwide and a major public health problem in all countries. ${ }^{55}$ In addition, the increasing occurrence of root caries in seniors was associated with the increasing occurrence of periodontitis. ${ }^{56}$ Root caries is commonly found in roots exposed to the oral environment as a progressive lesion, because of loss of periodontal attachment due to periodontitis which in turn favor root caries development. This could thus set off a vicious cycle of periodontal disease leading to root exposure leading to caries, requiring class $\mathrm{V}$ restorations whose subgingival margins would be difficult to clean and leave pockets for periodontal pathogen growth. Therefore, it would be highly desirable to develop a bioactive bonding agent for class $\mathrm{V}$ restorations with the cured bonding resin in subgingival margins to inhibit periodontal pathogens and provide the needed anti-periodontopathogenic function.

Biofilms are composed of a three-dimensional structure containing different bacterial species and extracellular polymeric substances (EPS). For biofilm formation, the bacterial colonizers can be classified into three groups: initial, middle, and late colonizers. In the present study, an in vitro periodontal biofilm model was performed using periodontitis-related species simulating the composition of in vivo subgingival plaque. The selected species are frequently found in the subgingival plaque $\mathrm{e}^{6,9,11,13}$ and include initial ( $S$. gordonii, $A$. naeslundii), intermediate ( $F$. nucleatum) and late colonizers $(P$. gingivalis), according to a previous study. ${ }^{53}$ Streptococci in particular recognize receptors in the salivary pellicle, which coats enamel immediately after the surface is cleaned. S. gordonii was included because streptococci accounts for $60-90 \%$ of initial colonizers of tooth surfaces. ${ }^{57}$ A. naeslundii represents $27 \%$ of the pioneer strains and is able to adapt to extreme fluctuating conditions, which is necessary for a pioneer bacteria strain to attach to dental surfaces. ${ }^{7} P$. gingivalis was frequently found in disease sites, suggesting that $P$. gingivalis plays an important role in the pathogenicity of periodontal diseases. ${ }^{12}$ In addition, $P$. gingivalis was frequently detected in later phase of subgingival biofilm formation. Therefore, these four species were selected and combined to form multi-species periodontal biofilm on resins. 
Saliva-derived protein adsorption on material's surface in vivo is a prerequisite for bacteria attachment. ${ }^{57}$ Therefore, making a protein-repellent resin would help repel bacterial attachment. Previous studies reported that most proteins adsorb preferentially onto hydrophobic surfaces. ${ }^{58}$ MPC is a methacrylate with phospholipid polar group in the side chain. $^{38}$ Phospholipids are a major component of all cell membranes as they can form lipid bilayers. ${ }^{59}$ MPC polymers are highly hydrophilic and well known to reduce protein adsorption and bacterial adhesion. ${ }^{\mathbf{3 8 - 4 0}}$ Regarding the protein-repellent mechanism, it was reported that, in the hydrated MPC polymer, there is an abundance of free water but no bound water. ${ }^{33}$ The bound water would result in protein adsorption. ${ }^{39,60}$ The large amount of free water around the phosphorylcholine groups could detach proteins, thereby repelling protein adsorption. ${ }^{39,60}$ The present study showed that incorporating $5 \%$ of MPC into the bonding agents reduced protein adsorption by more than an order of magnitude, from $5.4 \mu \mathrm{g} \mathrm{cm} \mathrm{cm}^{-2}$ on control resin to $0.36 \mu \mathrm{g} \mathrm{cm}^{-2}$ on the resin containing MCP. This indeed led to much less single and multi-species periodontal biofilm formation on the resin.

Furthermore, the present study showed that combining MPC with DMAHDM achieved the greatest inhibition of periodontal biofilms. For example, the multi-species periodontal biofilm CFU (per disk) was approximately $10^{9}$ on control disks; it was reduced to $10^{8}$ via MPC, to $10^{7}$ via DMAHDM, and finally to $10^{6}$ via MCP + DMAHDM. The mode of antibacterial action for QAMs is suggested to be contact-inhibition. ${ }^{31}$ The positivelycharged quaternary amine $\mathrm{N}^{+}$interacts with the negativelycharged cell membrane of bacteria, leading to membrane disruption and cytoplasmic leakage. ${ }^{31}$ This contact-killing mechanism would indicate that, when a salivary protein pellicle separates the antibacterial resin from the overlaying biofilm, the antibacterial effects of the resin could be reduced. ${ }^{31}$ However, because MPC could repel proteins from covering the resin surface, it would lead to direct contact of resin surface with bacteria, thus enhancing the contact-killing efficacy. Therefore, DMAHDM and MPC could work synergistically to maximize the periodontal bacteria inhibition efficacy.

The different types of biofilms showed different degrees of difficulty to be inhibited by the DMAHDM-MPC bonding agent. The biofilm CFU, MTT and polysaccharide level results indicate that the multi-species periodontal biofilm was less sensitive than $P$. gingivalis single-species biofilm to DMAHDM-MPC resin. Previous studies did not find significant difference in killing efficacy by QAMs against saliva microcosm biofilm and $S$. mutans single-species biofilm. ${ }^{58,59}$ Another study also found no difference in antibiotic resistance between multi-species bacteria and $S$. gordonii bacteria. ${ }^{60}$ However, studies showed an increased resistance to antibacterial agents in multi-species biofilms than single species biofilm. ${ }^{61}$ Multi-species biofilm cells produce extracellular polymeric substances (EPS), which is composed of polysaccharides, proteins and extracellular DNA, which hold them together and favor a three-dimensional spatial arrangement. ${ }^{62}$ The protective function of the matrix may be associated with specific components produced by one species that benefit the whole population. Zollinger et al. demonstrated that antibacterial effects of taurolidine were substantially reduced in a 12-species multispecies biofilm compared with single-species biofilm. ${ }^{63}$ Previous study showed that F. nucleatum coaggregated with $P$. gingivalis, and both species coaggregated with $S$. gordonii, playing a synergistic effect. ${ }^{9}$ Therefore, two bacterial mixtures of $P$. gingivalis and $F$. nucleatum resulted in greater survival than the monocultures. ${ }^{\mathbf{6 4}}$ It was also reported that $P$. gingivalis was capable of co-aggregating with $A$. naeslundii and $S$. gordonii. ${ }^{65}$ Interspecies aggregates may function as a synergistic micro-consortium that offers protection from shear stress and desiccation and thus contributes to biofilm accumulation, leading to the high resistance to antibacterial agents. Therefore, it was suggested that effective antimicrobial agents need to possess the ability to dissociate and kill aggregates in a short period of exposure time, to minimize biofilm re-establishment. ${ }^{66}$

Indeed, a previous study indicated that, when treated with $0.2 \%$ chlorhexidine, the CFU difference between single and multi-species biofilms was more than 2 log. ${ }^{67}$ Another study investigated the differences between single and dual species biofilms of Streptococcus mutans and Veillonella parvula in their susceptibility to chlorhexidine, and showed that the CFU reduction of $24 \mathrm{~h}$ biofilms was $2 \log .{ }^{68}$ Another study also indicated that $P$. gingivalis single-species biofilm was more sensitive to taurolidine than a multi-species (12-species) biofilm containing $P$. gingivalis, with the CFU difference being almost 3 $\log .{ }^{63}$ In the present study, the CFU difference between multispecies biofilm and $P$. gingivalis single-species biofilm was less than one log. A possible reason may be that the dual agents, MPC and DMAHDM, could repel saliva pellicles and directly kill the attached bacteria from the initial stage, thus achieving effective killing for all biofilms and resulting in a smaller difference between single and multi-species biofilms.

Polysaccharide is synthesized by live bacteria and is related to bacterial viability. In a biofilm, bacterial cells are surrounded by EPS which is composed of polysaccharides, proteins and extracellular DNA. EPS accounts for about $90 \%$ of the total biofilm mass. ${ }^{69}$ Polysaccharide synthesis by both multi-species and single-species biofilms in the present study was substantially reduced via the 5\% DMAHDM + 5\% MPC resin. The multispecies biofilm polysaccharide production was $86 \mathrm{mg} \mathrm{L}^{-1}$ for the control; it was reduced to $16 \mathrm{mg} \mathrm{L}^{-1}$ via MCP + DMAHDM. The single species biofilm polysaccharide production, using $P$. gingivalis as an example, was $70 \mathrm{mg} \mathrm{L}^{-1}$ for the control; it was reduced to $11 \mathrm{mg} \mathrm{L}^{-1}$ via MCP + DMAHDM. EPS with polysaccharide protects pathogens from antibacterial agents, and contributes to the virulence and pathogenicity of pathogens via small molecule-mediated inter- and intra-species crosstalks. ${ }^{64}$ Microbes would decelerate the synthesis of EPS when suffering from external stresses, since more energy is needed for their own survive. Therefore, the reduction in ESP via DMAHDM and MPC could reduce the protection that the bacteria have, and lessen the toxicity of the pathogens and potentially inhibit local periodontitis.

Besides the capability to kill periodontal pathogens, bonding to the tooth structure is also critically important for the restoration. This is because the bonded interface is the weak link of 
the restoration, and a de-bonded interface would allow food debris and bacteria invasion. Modification of the bonding agent via incorporation of bioactive agents should ensure that the bond strength is not compromised. Indeed, when $5 \%$ DMAHDM and 7.5\% MPC were both incorporated, the dentin bond strength decreased to $17 \mathrm{MPa}$ from $23 \mathrm{MPa}$ for the control. However, when the MPC content was reduced to $5 \%$, the addition of MPC and DMAHDM caused no significant decrease in the dentin bond strength. Nonetheless, further study is needed to investigate the long-term durability of the bonded interface using MCP + DMAHDM in tooth root cavities. In addition, the DMAHDM-MPC adhesive also contained 30\% NACP. The reason for incorporating NACP was for $\mathrm{Ca}$ and $\mathrm{P}$ ion release and remineralization of tooth lesions. ${ }^{41-43}$ A previous study showed that a NACP-containing dental composite effectively regenerated the lost minerals in enamel lesions, achieving a remineralization in enamel that was four-fold that of a commercial fluoride-releasing composite. ${ }^{\mathbf{1 1}}$ In an in situ study using human participants, the NACP composite successfully suppressed caries formation at the enamel-composite margins under oral biofilm acid attacks. ${ }^{42}$ Therefore, in a class $\mathrm{V}$ restoration, NACP is expected to help remineralize root lesions and inhibit recurrent caries. Further studies are needed to investigate the protection of class $\mathrm{V}$ restorations and the remineralization and strengthening of tooth roots via the DMAHDM-MPC-NACP method. Further studies should also investigate the application of the novel DMAHDM-MPC-NACP composition to other preventive and restorative dental materials such as sealants, composites, cements and tooth root coatings.

\section{Conclusions}

This study developed a novel bioactive bonding agent for class $\mathrm{V}$ restorations with a combination of protein-repellent and antibacterial capabilities to combat periodontal biofilms. The effects of MPC + DMAHDM in PEHB adhesive containing remineralizing NACP on dentin bond strength, protein adsorption, and periodontitis-related single and multi-species biofilms were determined for the first time. Dentin bond strength with 5\% DMAHDM and 5\% MPC were similar to that of a commercial control. Bonding agent with 5\% DMAHDM showed a strong antibacterial function. Bonding agent with 5\% MPC was strongly protein- and bacteria-repellent. The use of dual agents, 5\% DMAHDM + 5\% MPC, achieved the greatest reduction in multispecies biofilm growth, metabolic activity and polysaccharide production. The novel resin achieved a greater killing efficacy against $P$. gingivalis single-species biofilm than against multispecies biofilm. However, the multi-species biofilm CFU was still reduced by 3 orders of magnitude. Therefore, the new bonding agent with DMAHDM and MPC is promising for class V restorations with subgingival margins to inhibit periodontal biofilms, combat periodontitis and protect the periodontium.

\section{Acknowledgements}

We thank Drs Xianju Xie, Kunneng Liang and Mary Anne S. Melo for fruitful discussions and help. This work was supported by NIH R01 DE17974 (HX), National Science Foundation of China NSFC 81400487 (LW), Youth Fund of Science and Technology of Jilin Province 20150520043JH (LW), China Postdoctoral Science Foundation 2015M581405 (LW), Health Department Research Projects in Jilin Province 2016Q032 (LW), 20165074 (CL), NSFC 81400540 (KZ), Beijing Municipal Administration of Hospitals' Youth Program QML20151401 (KZ), University of Maryland School of Dentistry bridging fund (HX), and University of Maryland seed grant (HX).

\section{References}

1 S. Griffin, P. Griffin, J. Swann and N. Zlobin, J. Dent. Res., 2004, 83, 634-638.

2 S. Shen, L. P. Samaranayake and H. K. Yip, J. Dent., 2004, 32, 667-678.

3 P. Hellyer, D. Beighton, M. Heath and E. Lynch, Br. Dent. J., 1990, 169, 201-206.

4 N. Beyth, A. J. Domb and E. I. Weiss, J. Dent., 2007, 35, 201206.

5 S. Filoche, L. Wong and C. Sissons, J. Dent. Res., 2010, 89, 818.

6 M. Quirynen, R. Vogels, W. Peeters, D. Steenberghe, I. Naert and A. Haffajee, Clin. Oral Implants Res., 2006, 17, 25-37.

7 J. Li, E. J. Helmerhorst, C. W. Leone, R. F. Troxler, T. Yaskell, A. D. Haffajee, S. S. Socransky and F. G. Oppenheim, J. Appl. Microbiol., 2004, 97, 1311-1318.

8 B. Nyvad and M. Kilian, Eur. J. Oral Sci., 1987, 95, 369-380.

9 P. E. Kolenbrander, R. N. Andersen, D. S. Blehert, P. G. Egland, J. S. Foster and R. J. Palmer, Microbiol. Mol. Biol. Rev., 2002, 66, 486-505.

10 L. A. Ximénez-Fyvie, A. D. Haffajee and S. S. Socransky, J. Clin. Periodontol., 2000, 27, 722-732.

11 E. Andrian, D. Grenier and M. Rouabhia, J. Dent. Res., 2006, 85, 392-403.

12 P. S. Kumar, A. L. Griffen, M. L. Moeschberger and E. J. Leys, J. Clin. Microbiol., 2005, 43, 3944-3955.

13 B. Signat, C. Roques, P. Poulet and D. Duffaut, Curr. Issues Mol. Biol., 2010, 13, 25-36.

14 C. D. Lynch, R. J. McConnell and N. H. Wilson, J. Dent. Educ., 2007, 71, 430-434.

15 J. L. Ferracane, Dent. Mater., 2011, 27, 29-38.

16 Y. Wei, N. Silikas, Z. Zhang and D. C. Watts, Dent. Mater., 2011, 27, 259-266.

17 P. Spencer and Y. Wang, J. Biomed. Mater. Res., 2002, 62, 447456.

18 F. R. Tay and D. H. Pashley, Am. J. Dent., 2003, 16, 6-12.

19 J. Park, Q. Ye, E. Topp, A. Misra, S. L. Kieweg and P. Spencer, Dent. Mater., 2009, 25, 1569-1575.

20 J. L. Ferracane, U. Lohbauer and W. M. Palin, Int. J. Adhes. Adhes., 2016, 69, 2-14.

21 R. Belli, S. Kreppel, A. Petschelt, H. Hornberger, A. R. Boccaccini and U. Lohbauer, J. Mech. Behav. Biomed. Mater., 2014, 37, 100-108.

22 V. C. B. Leitune, F. M. Collares, R. M. Trommer, D. G. Andrioli, C. P. Bergmann and S. M. W. Samuel, J. Dent., 2013, 41, 321-327. 
23 K. Hikita, B. Van Meerbeek, J. De Munck, T. Ikeda, K. Van Landuyt, T. Maida, P. Lambrechts and M. Peumans, Dent. Mater., 2007, 23, 71-80.

24 W. J. Dunn, Am. J. Orthod. Dentofacial Orthoped., 2007, 131, 243-247.

25 C. Chen, M. D. Weir, L. Cheng, N. J. Lin, S. Lin-Gibson, L. C. Chow, X. Zhou and H. H. K. Xu, Dent. Mater., 2014, 30, 891-901.

26 S. Imazato, A. Ehara, M. Torii and S. Ebisu, J. Dent., 1998, 26, 267-271.

27 F. R. Tay and D. H. Pashley, Am. J. Dent., 2003, 16, 6-12.

28 X. Xu, L. Ling, R. Wang and J. O. Burgess, Dent. Mater., 2006, 22, 1014-1023.

29 S. Imazato, Dent. Mater., 2003, 19, 449-457.

30 F. Li, M. Weir and H. Xu, J. Dent. Res., 2013, 92, 932-938.

31 N. Beyth, I. Yudovin-Farber, R. Bahir, A. J. Domb and E. I. Weiss, Biomaterials, 2009, 27, 3995-4002.

32 N. Namba, Y. Yoshida, N. Nagaoka, S. Takashima, K. Matsuura-Yoshimoto, H. Maeda, B. Van Meerbeek, K. Suzuki and S. Takashiba, Dent. Mater., 2009, 25, 424-430. 33 R. Müller, A. Eidt, K.-A. Hiller, V. Katzur, M. Subat, H. Schweikl, S. Imazato, S. Ruhl and G. Schmalz, Biomaterials, 2009, 30, 4921-4929.

34 K. Ishihara, T. Ueda and N. Nakabayashi, Polym. J., 1990, 22, 355-360.

35 K. Ishihara and Y. Iwasaki, J. Biomater. Appl., 1998, 13, 111127.

36 J. Sibarani, M. Takai and K. Ishihara, Colloids Surf., B, 2007, 54, 88-93.

37 N. Zhang, M. A. Melo, C. Chen, J. Liu, M. D. Weir, Y. Bai and H. H. Xu, Dent. Mater., 2015, 31, 1119-1131.

38 N. Zhang, M. D. Weir, E. Romberg, Y. Bai and H. H. Xu, Dent. Mater., 2015, 31, 845-854.

39 M. A. S. Melo, L. Cheng, M. D. Weir, R. C. Hsia, L. K. Rodrigues and H. H. Xu, J. Biomed. Mater. Res., Part $B, 2013$, 101, 620-629.

40 L. Zhang, M. D. Weir, G. Hack, A. F. Fouad and H. H. Xu, J. Dent., 2015, 43, 1587-1595.

41 M. Weir, L. Chow and H. Xu, J. Dent. Res., 2012, 91, 979-984.

42 M. A. S. Melo, M. D. Weir, L. K. Rodrigues and H. H. Xu, Dent. Mater., 2013, 29, 231-240.

43 H. H. Xu, J. L. Moreau, L. Sun and L. C. Chow, Dent. Mater., 2011, 27, 762-769.

44 J. M. Antonucci, D. N. Zeiger, K. Tang, S. Lin-Gibson, B. O. Fowler and N. J. Lin, Dent. Mater., 2012, 28, 219-228.

45 C. Zhou, M. D. Weir, K. Zhang, D. Deng, L. Cheng and H. H. Xu, Dent. Mater., 2013, 29, 859-870.

46 J. Antonucci, J. O'Donnell, G. Schumacher and D. Skrtic, J. Adhes. Sci. Technol., 2009, 23, 1133-1147.
47 S. Imazato, Y. Kinomoto, H. Tarumi, S. Ebisu and F. R. Tay, Dent. Mater., 2003, 19, 313-319.

48 K. Ishihara, N. P. Ziats, B. P. Tierney, N. Nakabayashi and J. M. Anderson, J. Biomed. Mater. Res., 1991, 25, 1397-1407.

49 D. Fteita, E. Könönen, E. Söderling and U. K. Gürsoy, Anaerobe, 2014, 27, 7-13.

50 M. Sánchez, A. Llama-Palacios, V. Blanc, R. Leon, D. Herrera and M. Sanz, J. Periodontal Res., 2011, 46, 252-260.

51 P. Tsaousoglou, S. Nietzsche, G. Cachovan, A. Sculean and S. Eick, J. Med. Microbiol., 2014, 63, 284-292.

52 R. Peyyala, S. Kirakodu, K. Novak and J. Ebersole, J. Dent. Res., 2013, DOI: 10.1177/0022034512472508.

53 M. Sánchez, A. Llama-Palacios, E. Fernández, E. Figuero, M. Marín, R. León, V. Blanc, D. Herrera and M. Sanz, Dent. Mater., 2014, 30, 1161-1171.

54 B. Yap, P. S. Zilm, N. Briggs, A. H. Rogers and P. C. Cathro, Aust. Endod. J., 2014, 40, 101-110.

55 P. E. Petersen and H. Ogawa, Periodontol. 2000, 2012, 60, 1539.

56 J. K. Kim, L. A. Baker, H. Seirawan and E. M. Crimmins, Spec. Care Dentist., 2012, 32, 234-241.

57 S. Periasamy and P. E. Kolenbrander, J. Bacteriol., 2009, 191, 6804-6811.

58 L. Cheng, K. Zhang, M. D. Weir, H. Liu, X. Zhou and H. H. Xu, Dent. Mater., 2013, 29, 462-472.

59 K. Zhang, L. Cheng, S. Imazato, J. M. Antonucci, N. J. Lin, S. Lin-Gibson, Y. Bai and H. H. Xu, J. Dent., 2013, 41, 464474.

60 J. H. Park, J. K. Lee, H. S. Um, B. S. Chang and S. Y. Lee, J. Periodontal Implant Sci., 2014, 44, 79-84.

61 M. Simoes, L. C. Simões and M. J. Vieira, Water Res., 2009, 43, 229-237.

62 S. Stepanović, D. Vuković, I. Dakić, B. Savić and M. ŠvabićVlahović, J. Microbiol. Methods, 2000, 40, 175-179.

63 L. Zollinger, S. Schnyder, S. Nietzsche, A. Sculean and S. Eick, Anaerobe, 2015, 32, 18-23.

64 D. Polak, A. Wilensky, L. Shapira, A. Halabi, D. Goldstein, E. I. Weiss and Y. Houri-Haddad, J. Clin. Periodontol., 2009, 36, 406-410.

65 S. C. Holt and J. L. Ebersole, Periodontol. 2000, 2005, 38, 72122.

66 S. K. Filoche, M. Zhu and C. D. Wu, J. Dent. Res., 2004, 83, 802-806.

67 J. Pratten, A. W. Smith and M. Wilson, J. Antimicrob. Chemother., 1998, 42, 453-459.

68 D. Kara, S. B. I. Luppens, J. Van Marle, R. Özok and J. M. Ten Cate, FEMS Microbiol. Lett., 2007, 271, 90-97.

69 M. T. Pöllänen, A. Paino and R. Ihalin, Int. J. Mol. Sci., 2013, 14, 17221-17237. 